\title{
Study into Dynamics of Structural Changes Occurring in Blood Plasma under Laser Radiation
}

\author{
Anna V. Neupokoeva*, Dmitry A. Gavrilov, and Andrey S. Yakutin \\ Department of Medical and Biological Physics, Irkutsk State Medical University, 1 Krasnogo Vosstaniya str., \\ Irkutsk 664009, Russian Federation
}

* e-mail: annett 2005@inbox.ru

\begin{abstract}
This paper focuses on studying the impact of laser irradiation $(\lambda=532 \mathrm{~nm}$, power $15 \mathrm{~mW})$ on human blood plasma. The structural changes in plasma were controlled by the crystallographic method with the mean crystallogram segment size as a numerical evaluation. In addition, the structural changes were explored by processing the speckle patterns obtained during laser radiation passes through plasma. Exposure to laser radiation of $\lambda=532 \mathrm{~nm}$ for $20 \mathrm{~min}$ resulted in the $30 \%$ reduced the crystallogram characteristic size with the dynamics of the structural changes coinciding with the dynamics discovered by speckle patterns processing. The crystallographic method revealed that laser induced structural changes in plasma remain for 24-36 h after exposure. (C) 2021 Journal of Biomedical Photonics \& Engineering.
\end{abstract}

Keywords: laser biostimulation; blood plasma; crystallogram; wavelet analysis; correlation image processing; speckle pattern.

Paper \#3404 received 20 Jan 2021; revised manuscript received 3 Mar 2021; accepted for publication 20 Mar 2021; published online 30 Mar 2021. doi: 10.18287/JBPE21.07.010302.

\section{Introduction}

Laser irradiation is widely used in medical practice. Depending on the radiation properties, e. g. wavelength, intensity, it can have both adverse (tissue destruction, burns) and beneficial effects on the body (reparative process stimulation, reduced tissue swelling and inflammation, improved tissue metabolism) [1].

However, little research has been conducted on the mechanism of laser impact both locally and systemically. Recently there have been a number of studies suggesting that laser radiation interacts with blood proteins changing their structure and functional activity [2, 3, 4]. According to this hypothesis, laser radiation is able to interact with the proteins within blood cell elements (erythrocytes, leukocytes, etc.) as well as plasma proteins. However, the effect of laser radiation on the cell-free biological media has not been adequately researched. Its possible effect on the human blood plasma structure can be beneficial and find practical use in transfusion therapy.

Nevertheless, the question of the stability level of structure changes remains open. It means that after some time the proteins partially restore the supramolecular structures when the relaxation process takes place. Studying the temporal characteristics of this process would make it possible to improve the frequency of laser procedures when treating patients.

On the other hand, the advent of new methods of examining structural changes in fluids $[5,6]$ has opened opportunities for a more detail research into the changes occurring during laser radiation in fluids. For this reason, the aim of this paper is to study the dynamics of structural changes occurring in blood plasma undergoing laser radiation as well as investigation of the stability margin of these changes over time.

\section{Experimental study}

\subsection{Materials and methods}

Human plasma was provided by Irkutsk Region Blood Transfusion Station (this organization holds the license of the Ministry of Healthcare of the Russian Federation for banking, storage and managing blood and its components as well as for operations in transfusiology). Human blood plasma was provided frozen. Before the experiments, plasma was allowed to thaw at room temperature. The source of laser radiation was a $303 \mathrm{LXi}$ diode-pumped solid-state laser (made in China) with a $532 \mathrm{~nm}$ wavelength. The source power was reduced by 
means of a neutral light filter to $15 \mathrm{~mW}$ with the intensity of $50 \mathrm{~mW} / \mathrm{cm}^{2}$. The threshold intensity for intravenous blood laser radiation is $1-1.5 \mathrm{~mW} / \mathrm{cm}^{2}$, whereas the maximum intensity for biostimulation is about $100 \mathrm{~mW} / \mathrm{cm}^{2}$. The latter correlates with the sunlight intensity on a clear day [4]. Therefore, the mean intensity was chosen for the experiment. We also took into account the fact that plasma absorption is lower than that of whole blood. The choice of the wavelength for laser irradiation was determined by the following: most methodologies of laser irradiation make use of the red and infrared range. Even though the last ten years have seen the advent of specialized medical devices using the $532 \mathrm{~nm}$ wavelength [7] that is well absorbed by haemoglobin, the effect of the laser radiation within the green range remains underinvestigated [4].

Interaction of laser radiation with protein macromolecules can involve several processes: destabilization of the macromolecule hydration shell, change in the secondary and tertiary protein structure, change in the mobility of macromolecule subunits and their density, as well as dissolution of multi-molecule clusters into smaller formations. Most of these processes should result in the change of the inhomogeneity characteristic size in a solution.

When the solution dries up, the first to fasten on the substrate are large macromolecule clusters (having a lower random velocity as a unit) that also act as nuclei (or crystallization centres) for crystal growing in the film. After that, epitaxial growth is taking place for lowmolecular fractions that later crystallize the surface of the dendrite-like backbone. Thus, a beam structure is built of the lamellas with epitaxial recrystallization on the beam section that acts as a substrate for the drying solution. Reduced lamellar beam thickness after laser irradiation demonstrates that in the irradiated solution the cluster sizes are reducing considerably, which results in nuclei size reduction during film drying [8].

The experimental study consisted of two stages: the first stage was investigating structural changes in blood plasma depending on laser exposure time; the second stage was investigating the stability of structural changes depending on the time after laser irradiation stopped.

At each stage, four sets of experiments were conducted. First, each set of experiments involved selecting blood plasma samples that had not been subjected to radiation. Then the plasma was placed into a cuvette and subjected to laser radiation. After 1, 2, 5, 10 and $20 \mathrm{~min}$ a $1 \mathrm{ml}$ blood plasma sample was taken from the cuvette. The sample was poured on the slide. After drying, the sample was examined under a microscope at low magnification $(10 \times)$ to record the obtained segments for each sample. Later on, calculations were made to define the statistical significance of the changes in a number of segments after exposure.

At the second stage, the radiated plasma in the cuvette was placed into a thermostat (at the temperature of $37^{\circ} \mathrm{C}$ ) to simulate physiological conditions. Further, the contents were poured on the substrate again 24, 36 and $48 \mathrm{~h}$ after laser exposure.
Along with sample collection, to obtain crystallograms at temporal control points, recordings were made of the speckle pattern obtained during laser radiation passing through the plasma cuvette. The installation diagram is shown in Fig. 1: laser radiation when passing through the cuvette with the test fluid scattered forming a speckle pattern on the recorder (CCD camera - Genius iLook 320). As is well-known, the speckle size on the speckle pattern is inversely related to the characteristic inhomogeneity size in the exposed medium. That is why changes in the speckle characteristic size reflect the changes in the inhomogeneity size in the test fluid.

To assess the speckle size, the wavelet analysis method was used. A section of $400 \times 400$ pixels was cut out of the speckle pattern, then a diagonal data line was selected on this section for wavelet transform. As the mother wavelet, the Morlet wavelet was used $[9,10]$.

Wavelet analysis makes it possible to determine the signal hidden periodicity. If a signal is a dependence of some parameter on time, the wavelet transform is able to determine not just the frequency but the characteristic change period of this process (a so-called time scale) as well as localization of this scale over time. If the test signal is the amplitude dependence on the spatial coordinates (as with our case), the wavelet analysis demonstrates the characteristic spatial period, i.e. the spot size of the speckle pattern. The results of the wavelet transform are presented as a skeleton: along the $\mathrm{x}$-axis are the pixels of the original speckle pattern, along the y-axis is the spatial scale, i.e. a characteristic speckle size (also in pixels), the amplitude is marked in colour (black is zero, white is one).

A true advantage of wavelet processing of speckle patterns is its speed and visual clarity. A limitation of this method is that it is able to process only one line of the whole speckle pattern, which can result in the loss or distortion of the information contained in the whole speckle field. The success of the wavelet analysis is also considerably dependent on the choice of the mother function - the more it is symbatic to the intensity distribution in the speckle spot the better is the result.

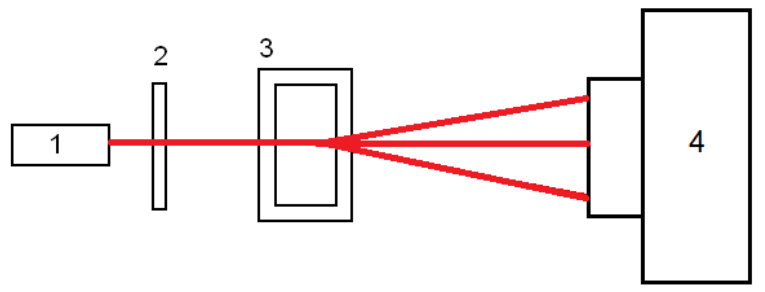

Fig. 1 Scheme of obtaining speckle patterns: laser radiation from source 1 is passing through light filter 2 and gets to fluid cuvette 3 where it interacts with structure inhomogeneities and forms a speckle pattern which is recorded by CCD camera 4 .

An alternative to the wavelet analysis method is calculating the coefficient of correlation between the test image and a regular structure of the chessboard type with a variable size of squares. The result of calculating the 
correlation field is the array of the correlation coefficients that can be presented graphically as a surface with a number of peaks. The bigger the coefficient of autocorrelation the higher is the peak $[5,6,11]$.

It is worth mentioning that if the square size and the speckle characteristic size coincide, the correlation peaks of regular conical shape are alternating at regular intervals. If the chessboard square size and the speckle size do not coincide, the correlation peaks will deform and become double-humped or concatenate $[6,11]$.

\subsection{Experimental data}

Crystallographic method of evaluating the inhomogeneity size has revealed that under laser radiation an oscillation behaviour took place. Drastic decrease in the segment size at short exposure times with further increase in the segment size (till the fifth minute) and then a smoother decrease (Fig. 2 demonstrates the averaged graph of changes for all sets). Statistical processing of the results of all 4 sets has demonstrated that these changes are not accidental. The crystallogram segment size decrease reaches $50 \%$ whereas the measurement error is $18-35 \%$ depending on the particular time point. Moreover, the behaviour (decrease - increase - decrease) was similar for all the sets, even though it had a different absolute value in each set.

The results of wavelet analysis for the diagonal line of the speckle patterns are shown in Fig. 3: along the $\mathrm{x}$-axis are the pixels of the original speckle pattern. Along the $y$-axis is the spatial scale, i.e. a characteristic speckle size in pixels. The amplitude is marked in colour (black is zero, white is one).

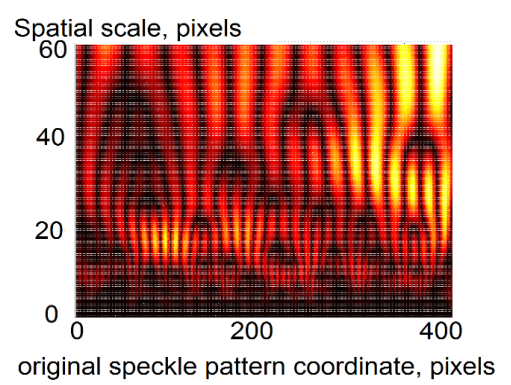

(before exposure)

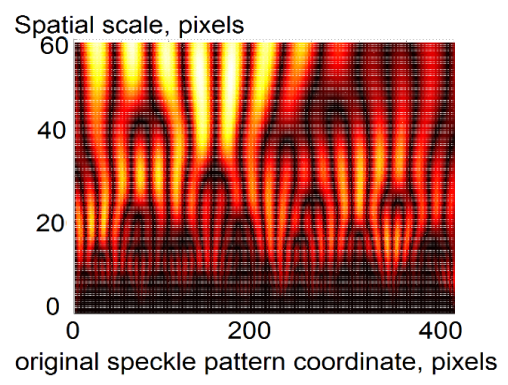

(after $10 \mathrm{~min}$ )
Analysis of Fig. 3 shows that before laser exposure, two series of maximums are observed. For the 20 pixel scale and for the 30 pixel scale, which corresponds to the actual speckle size of 125-190 $\mu \mathrm{m}$. After 5 min exposure, a clear series of maximums was defined (the speckle size is about $250 \mu \mathrm{m}$ ), however, at the same time the smallscale component of about 10-15 pixels (the presence of 60-90 $\mu \mathrm{m}$ speckles) had comparatively large amplitudes, which indicates a considerable instability of inhomogeneity sizes in the test solution. Further extending the time of laser exposure, increases the contribution of the large-scale components, which reflects the decrease in the inhomogeneity size in plasma. In general, it would be safe to say that the speckle characteristic size increases from 20-30 to 30-40 pixels, which corresponds to the actual change of the speckle size from $125-190 \mu \mathrm{m}$ to $190-250 \mu \mathrm{m}$, i.e. 1.5-2 times.

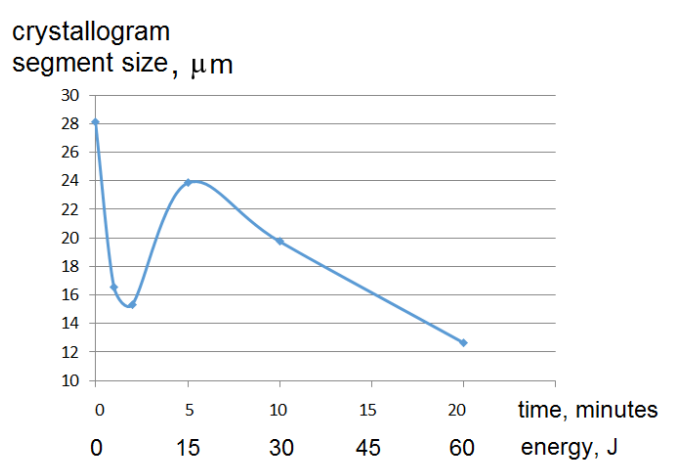

Fig. 2 Changes in the crystallogram segment average size depending on the time of blood plasma laser exposure: along the $y$-axis is the crystallogram segment size in $\mu \mathrm{m}$.

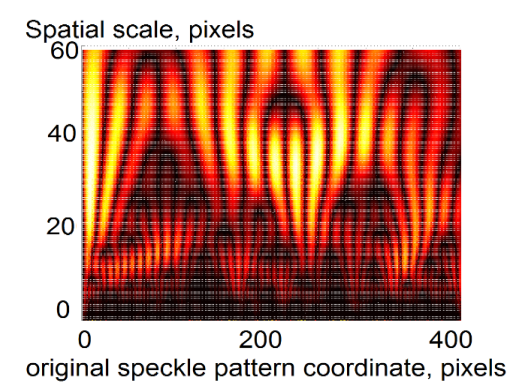

(after $5 \mathrm{~min}$ )

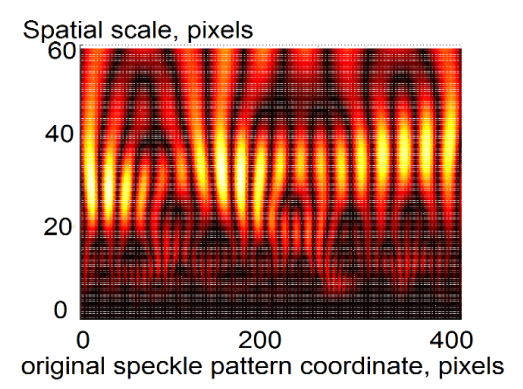

(after $20 \mathrm{~min}$ )

Fig. 3 The results of wavelet analysis of the diagonal line of the speckle pattern depending on the time of blood plasma laser exposure. 


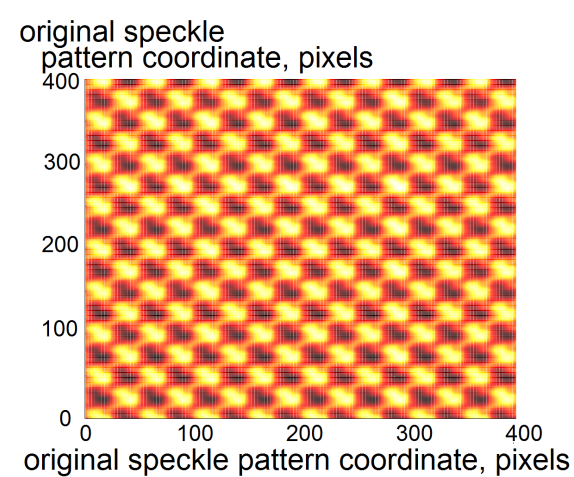

(before exposure)

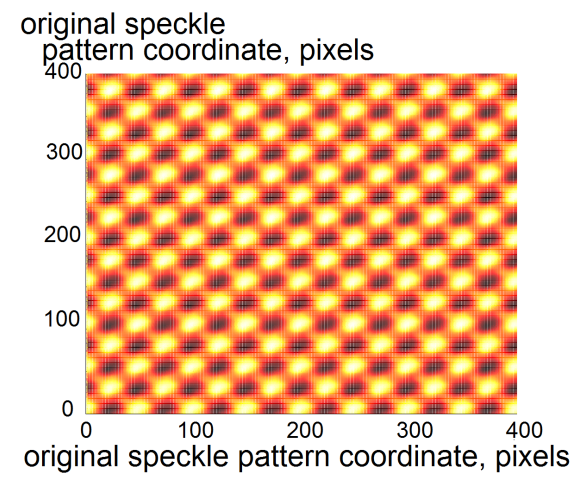

(after $10 \mathrm{~min}$ )

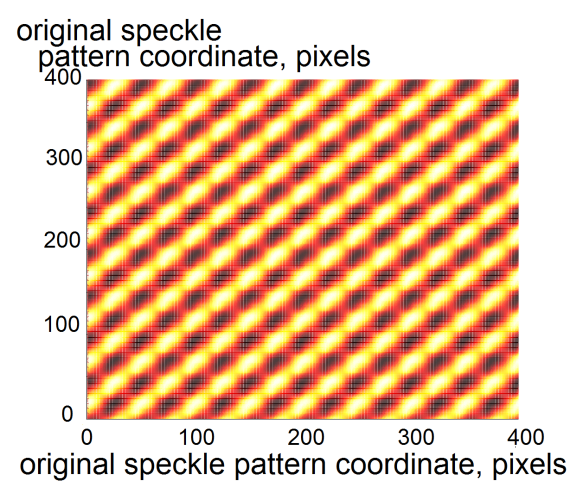

(after 5 min)

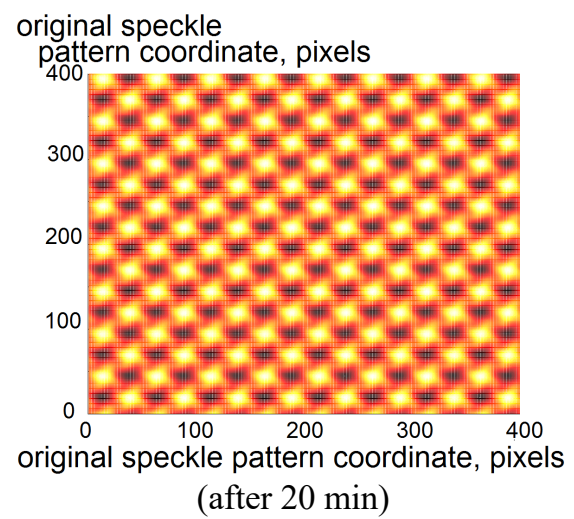

Fig. 4 The results of calculating the correlation coefficient between the speckle pattern and a regular structure of the chessboard type with the square size of $25 \times 25$ pixels. Along the axes are the pixels of the original speckle pattern, the correlation coefficient amplitude is marked with colour: black is zero, white is one.

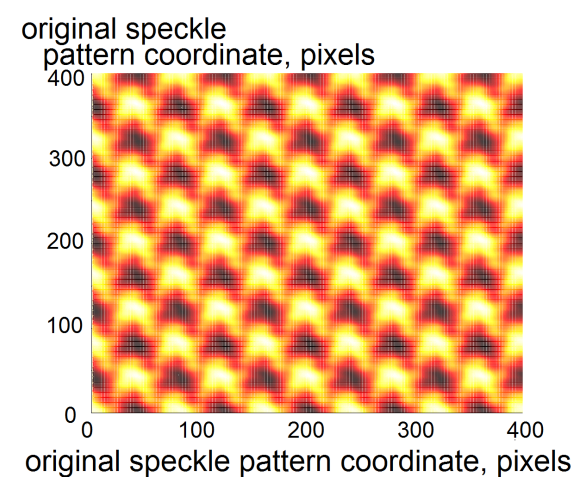

(before exposure)

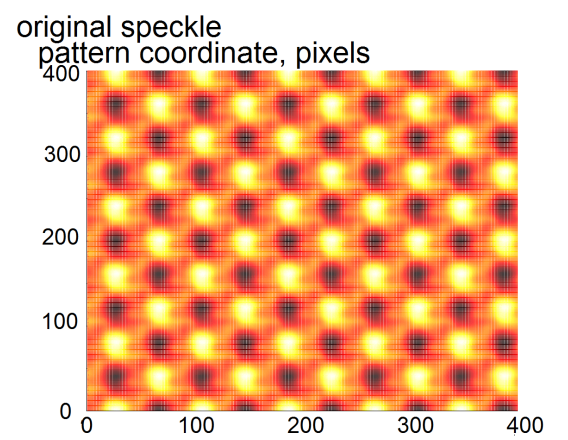

original speckle pattern coordinate, pixels (after $10 \mathrm{~min}$ )

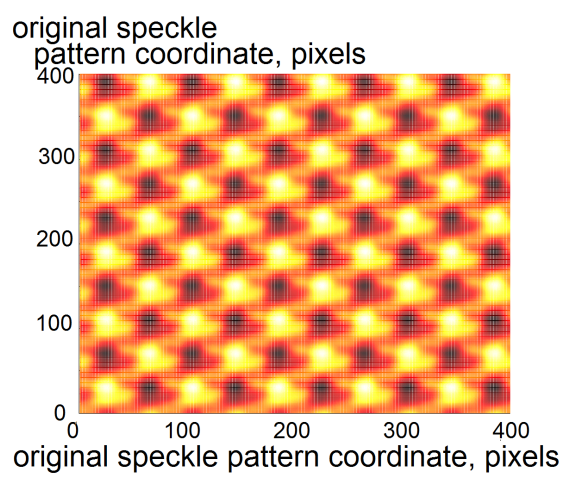

(after $5 \mathrm{~min}$ )

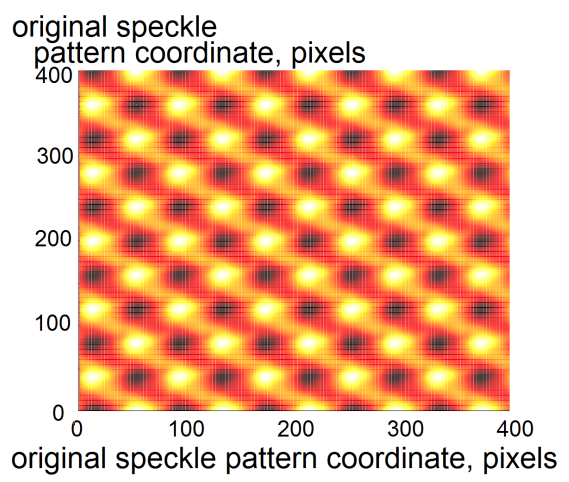

(after $20 \mathrm{~min}$ )

Fig. 5 The results of calculating the correlation coefficient between the speckle pattern and the regular structure of the chessboard type with the square size of $40 \times 40$ pixels. Along the axes are the pixels of the original speckle pattern, the correlation coefficient amplitude is marked with colour: black is zero, white is one. 
The results of calculating the coefficient of correlation between the speckle pattern and a regular structure of the chessboard type are shown in Figs. 4-5. For convolution 2 chessboards were selected: with the square size of $25 \times 25$ and $40 \times 40$ pixels. Calculating the correlation field results in the array of the correlation coefficients that can be presented graphically as a surface with a number of peaks. The bigger the coefficient of auto-correlation, the higher is the peak.

Coincidence of the speckle size with the chessboard square size forms a regular conical maximum whose top view is thus a clear round spot. Fig. 4 shows that the correlation field from the reference sample has characteristic twin maximums, which takes place when the speckle size is somewhat smaller than the chessboard square size. From the speckle pattern obtained after 5 min laser exposure, we can observe some joined correlation peaks, which is characteristic in cases where the speckle size is somewhat larger than the chessboard square size. The most pronounced correlation peaks for convolution with the chessboard of $25 \times 25$ pixels can be observed for the speckle pattern obtained after $10 \mathrm{~min}$ laser exposure. For the correlation pattern after $20 \mathrm{~min}$ exposure, the maximum is rather diamond-shaped because there is a complex maximum in the form of the central peak with 4 additional maximums at its base. Such picture emerges when the speckle size does not agree with the size of the chessboard square size.

Analysis of the correlation fields shown in Fig. 5 demonstrates that before the exposure and at short ( $5 \mathrm{~min}$ ) exposures, the characteristic speckle size is smaller than the chessboard square. This results in considerable deformation of the correlation peaks and emergence of the twin peak. With the increase in the laser exposure time to $10 \mathrm{~min}$, the correlation maximums obtain a regular form indicating the agreement between the characteristic speckle size and the chessboard square size ( 40 pixels). In the case of the correlation field for the speckle pattern obtained after 20 min plasma irradiation, the maximums concatenate. This reflects further increase in the speckle characteristic size.

Thus, the method of calculating the correlation coefficient between the speckle pattern and the regular chessboard-type structure has shown that the characteristic speckle size is gradually increasing, which reflects the decrease in the inhomogeneity size and is in good agreement with the results obtained by the crystallography method.

The second stage of the experiment was aimed at the investigation of the relaxation processes and determining the time during which the structural changes caused by a single laser exposure remain in blood plasma. Investigating relaxation processes in protein solution [12] has shown that they lead to gradual increase in the protein macromolecule cluster sizes in a solution with the structural changes caused by laser radiation remaining for no longer than $24 \mathrm{~h}$. On the strength of these data we suggested that in blood plasma the relaxation processes should proceed in a similar way. However, taking into account a more diversified composition of protein components in plasma compared to the model solution, we assumed that the processes of restoring the primary plasma structure should proceed slower, therefore the observation time was prolonged to $48 \mathrm{~h}$. The results of investigating the plasma structural changes by the crystallography method are shown in Fig. 6 (the averaged data of 4 sets).

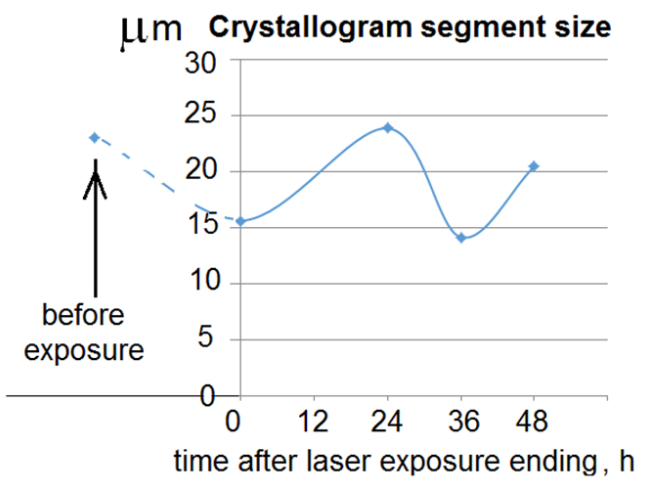

Fig. 6 Changes in the crystallogram segment average size depending on the time after laser exposure to blood plasma: along the y-axis is the crystallogram segment size in $\mu \mathrm{m}$.

The experiment has revealed that the changes in plasma structure caused by laser exposure have an oscillation character that took place in all the 4 sets of the experiment. The measurement error was $6-17 \%$, which is considerably less than the relative amount of change that was $30-50 \% .24 \mathrm{~h}$ after the exposure starts, the system returns to the non-irradiated state. However, then the structure characteristic size decreases again and completely resets to the non-irradiated state no earlier than $48 \mathrm{~h}$ after the laser exposure ending.

\section{Discussion}

Since plasma is a cell-free protein solution, it is obvious that the target of laser exposure are proteins. In our opinion, there may be two different scenarios of interaction of laser radiation with protein molecules.

As is well-known, at different levels of protein molecule arrangement there are chemical bonds of different strengths: the primary structure has peptide bonds (covalent bonds, strong); the secondary structure has hydrogen bonds (weak); the tertiary structure has hydrogen hydrophobic interactions (weak), and sometimes disulphide bonds (strong); the quaternary structure (for certain proteins) has ion, hydrogen, hydrophobic interactions (weak). The energy of the laser radiation quantum in the employed range is too small to break the peptide or disulphide bonds (whose energy is hundreds of $\mathrm{kJ} / \mathrm{mole}$ ) but is sufficient to break the hydrogen bonds (whose energy is tens of $\mathrm{kJ} / \mathrm{mole}$ ) or to destabilize the hydrophobic section because in these cases the bond energy is an order of magnitude less than that with covalent interaction $[13,14]$. That is why laser radiation is capable of changing the secondary, tertiary and quaternary protein structures without affecting the 
primary structure, which results in both dissolved configuration of certain molecules and of larger supramolecular complexes into smaller ones.

The second scenario is related to the presence of the hydration shell around the protein molecule. Consequently, laser radiation capable of energizing a protein molecule, is able to destabilize the hydration shell of the protein molecules making structural changes easier. Moreover, the radiation is capable of disturbing the thermodynamic equilibrium of the protein and breaking the weak bonds inside the molecule at the secondary and/or tertiary organisation level thus causing structural changes [14].

Refs. $[2,15]$ revealed the influence of laser radiation on the secondary and tertiary structures of blood proteins with blood haemoglobin as the main laser radiation acceptor. It is noted that the changes detected in whole blood are much more pronounced than the changes in plasma. However, our findings demonstrate the presence of structural changes in plasma. Therefore, we believe that the role of plasma proteins in the response of living bodies to laser radiation is no less crucial than the role of blood cell components.

Ref. [16] suggests that pulsed magnetic field can cause conformational changes in the hydration shell of antibiotic molecules, which results in the changes of its activity. We believe that in the interaction between laser radiation and protein water solutions (which includes blood plasma), it is equally important to consider the role of the macromolecule hydration shell as well as the ability of the macromolecules themselves to make associates [17], which affects its functional activity.

Therefore, the interaction of laser radiation with plasma proteins can be described as follows. First, the proteins receive additional energy in the form of laser radiation, which changes the balance of interaction forces between the protein and its hydration shell and enables the proteins to release from it. Without hydration shell they start interacting between themselves and with the charged ions forming large conglomerates. Concurrently, structural changes of protein molecule are taking effect. Due to excess energy, weak bonds are breaking, protein is changing its structure and its activity is altering.

Thus, the oscillation changes in the structure characteristic size in plasma under the laser irradiation and in the relaxation process after laser exposure are connected with two contrary processes. On the one hand, destabilization of the hydration shell, increased mobility of certain parts of macromolecules and their charged groups lead to loosening of associates and possibility to interact with other protein molecules, thus making large associates. On the other hand, macromolecules, when disengaged from the associate and due to extra energy, are able to convolute themselves in such a way that their dipole moment is equal to zero and their further interaction with other macromolecules is reduced to minimum. After the fifth minute, we can observe that the process of building small closely-packed protein formations is dominating over the process of building large associates.

Protein structural changes under laser radiation and may have a beneficial effect, because radiation itself does not typically cause serious damage. Some proteins will restore independently after a while, and, at the same time some proteins can get activated in the living system. For instance, laser radiation, being a stress factor, can facilitate heat-shock protein synthesis - a class of proteins synthesized when structural changes of normal protein molecules are detected and responsible for restoring the regular structure and assembly of other proteins; participating in the immune response [18, 19]. Therefore, changes in the functional activity of some proteins effect the structure and production of others, which, in total, ensures a complex immune response not only in the affected area but in the body as a whole.

\section{Conclusion}

When affected by laser radiation with $532 \mathrm{~nm}$ wavelength, blood plasma structure is changing and these changes have an oscillatory character: with a short exposure time (1-2 min), the characteristic structure size reduces, then begins to grow once more and after that (the exposure time over $10 \mathrm{~min}$ ) gradually decreases again. Various techniques of recording structural changes, e.g. by means of crystallograms and of processing speckle patterns obtained during laser radiation through plasma, have shown similar results: under laser radiation, blood plasma undergoes a reduction to a factor of 1.5-2 in the characteristic size of inhomogeneities. Oscillation changes of inhomogeneity size are likely explained by two competing processes related to the changes in protein molecules themselves and their hydration shells when blood plasma is exposed to laser radiation.

Examining the plasma structure relaxation processes after laser exposure has also revealed oscillation of the changes, which can call for further studies.

\section{Disclosures}

All authors declare that there is no conflict of interests in this paper.

\section{References}

1. A. V. Kartelishev, A. G. Rumyancev, A. R. Evstigneev, A. V. Geiniz, and S. V. Usova (Eds.), Lazernaya terapiya i profilaktika, Prakticheskaya meditsina, Moscow, Russia (2012) [in Russian]. ISBN 978-5-98811-219-8.

2. G. A. Zalesskaya, E. G. Sambor, "Interaction of low-intensity laser radiation with blood and its components," Journal of Applied Spectroscopy 72(2), 230-235 (2005) [in Russian]. 
3. A. N. Malov, A. Yu. Seteikin, A. V. Neupokoeva, E. S. Musatova, I. E. Golub, L. V. Sorokina, V. S. Fetschenko, and A. A. Vaichas, "The laser radiation action on the biological objects," Optik 124(23), 6034-6041 (2013).

4. S. V. Moskvin (Ed.), Osnovy lazernoy terapii. Seriya “Effektivnaya lazernaya terapiya,” Triada, Tver, Russia (2016) [in Russian]. ISBN 978-5-94789-738-8.

5. P. V. Pavlov, A. N. Malov, and A. V. Neupokoeva, "Determination of the working fluids technical condition by analysis of digital speckle images parameters," in Proceedings of VII International Conference on Photonics and Information Optics (PhIO), National Research Nuclear University Mephi, Moscow, 490-497 (2018) [in Russian].

6. A. V. Neupokoeva, A. N. Malov, "Comparative analysis of structural changes in fluid media monitoring methods," Izvestiya vyshih uchebnyh zavedeniy. Physica 58(11-3), 164-168 (2015) [in Russian].

7. A. V. Gheinits, S. V. Moskvin, and A. A. Anchilov, Intravenous laser blood irradiation, Triada, Tver, Russia (2012) [in Russian].

8. Y. Y. Tarasevich, "The models and mechanisms of the dehydration self-organization of biological fluids," PhysicsUspekhi 47(7), 717-728 (2004).

9. I. M. Dremin, O. V. Ivanov, and V. A. Nechitailo, "Wavelts and their uses," Physics-Uspekhi 44(5), 447-478 (2001).

10. N. M. Astaf'eva, "Wavelet analysis: basic theory and someapplications," Physics-Uspekhi 39(11), 1085-1108 (1996).

11. A. N. Malov, A. V. Neupokoeva, "A chessboard method for the speckle images analysis," in Collection of works of the 10th International Conference "GoloExpo 2013", Moscow, 257-264 (2013) [in Russian].

12. K. V. Rogova, E. R. Gerok, and A. V. Neupokoeva, "Investigation of the protein solution structure relaxation processes after laser exposure," in Proceedings of the XVIII All-Russian Youth Samara Competition-Conference of Scientific Papers on Optics and Laser Physics, P. N. Lebedev Physical Institute of the Russian Academy of Sciences, Moscow, 326-332 (2020) [in Russian].

13. N. L. Glinka, General chemistry, Integral-Press, Moscow, Russia (2003) [in Russian].

14. A. V. Finkelshtein, O. B. Ptitsyn (Eds.), Fizika belka, KDU, Moscow, Russia (2012) [in Russian].

15. G. A. Zalesskaya, I. I. Kalosha, "Effect of in vivo irradiation of blood by low-intensity emission from a He-Ne laser on the molecular components of blood," Journal of Applied Spectroscopy 76(5), 720-726 (2009).

16. V. A. Glushchenkov, T. I. Vasilyeva, I. A. Belyaeva, N. A. Rodenko, A. K. Madyarova, and R. J. Jusupov, “Changes in the antibacterial activity of benzylpenicillin exposed to a pulsed high-intensity magnetic field," Biophysics 64(2), 214-223 (2019).

17. V. D. Lakhno (Ed.), Klastery v fizike, khimii, biologii, Regulyarnaya i khaoticheskaya dinamika, Izhevsk, Russia (2001) [in Russian]. ISBN 5-93972-060-9.

18. M. Nishikawa, S. Takemoto, and Y. Takakura, "Heat shock protein derivatives for delivery of antigens to antigen presenting cells," International Journal of Pharmaceutics 354 (1-2), 23-27 (2008).

19. S. Walter, J. Buchner, "Molecular chaperones - cellular machines for protein folding," Angewandte Chemie International Edition 41(7), 1098-1113 (2002). 